\title{
Small-Bowel Hemangiosarcoma and Capsule Endoscopy
}

Capsule endoscopy is emerging as a sensitive technique for evaluating chronic gastrointestinal bleeding, and it appears to have better diagnostic yields than push enteroscopy, for example [1]. Bleeding sites in the small bowel are often difficult to detect using conventional radiological or scintigraphic techniques, and this sometimes makes laparotomy or surgical intervention necessary $[2,3]$. With capsule endoscopy, it is now possible to visualize small-intestinal bleeding sites beyond the range of regular endoscopes [4]. We present here the first capsule endoscopy images of a small-bowel hemangiosarcoma causing chronic gastrointestinal bleeding.

A 72-year-old man who had undergone several inconclusive diagnostic procedures, including upper and lower endoscopies, was referred with an 18-month history of bleeding and anemia, requiring several blood transfusions and hospital referrals due to abdominal pain. Capsule endoscopy (Given M2A, Given Imaging Ltd., Yoqneam, Israel) was carried out to evaluate possible bleeding sites in the small bowel. The images were reviewed independently by two persons experienced in viewing and interpreting standard endoscopic images, and revealed normal findings (Figure 1a), with the exception of dark, bloody liquid in the distal part of the small bowel (Figure 1b). An ex-
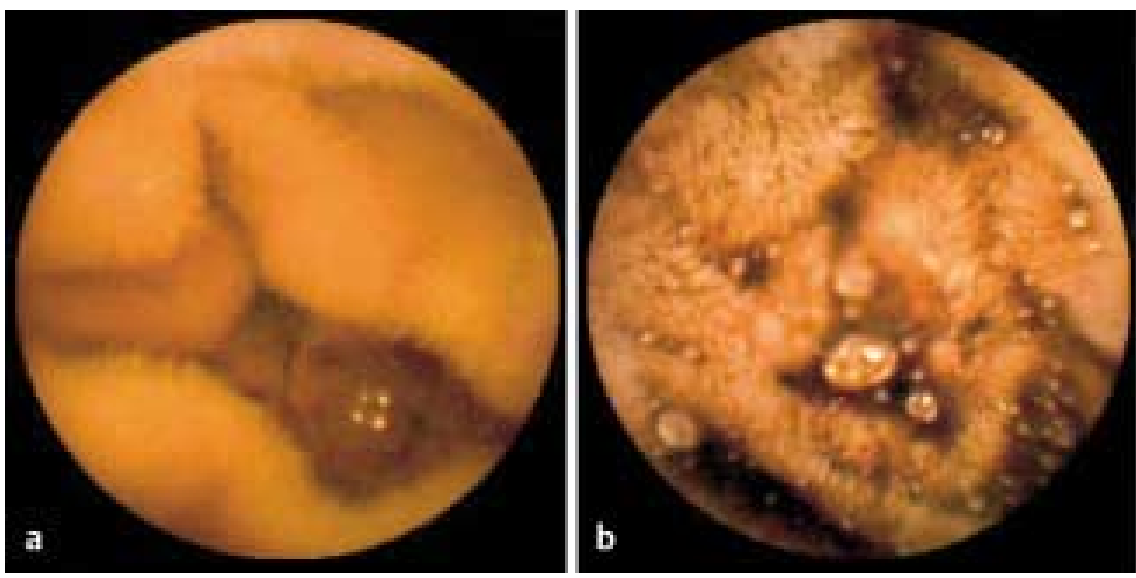

ploratory laparotomy revealed that a small tumor $(10 \times 10 \times 7 \mathrm{~mm}), 70 \mathrm{~cm}$ beyond the ligament of Treitz, was the primary bleeding site. Microscopy and immunohistochemistry identified it as an intestinal hemangiosarcoma, which often presents in a multifocal pattern [5]; for this reason, another capsule endoscopy, with normal findings, was carried out 5 weeks later. A retrospective review of the first capsule endoscopy clearly showed the tumor as a protruding mass (Figure 1c).

This case illustrates the technical superiority of capsule endoscopy as a diagnostic tool for clarifying small-bowel tumors, but also shows that the sensitivity of capsule endoscopy may depend on the reviewing process and the reviewer. For this reason, it should be emphasized that endoscopists who are still on the learning curve in capsule endoscopy need to remain alert. In addition, the case shows that capsule endoscopy is an obvious follow-up modality after treatment of smallbowel diseases associated with frequent recurrence or multifocal presentation.

\section{F. K. Knop, M. B. Hansen, S. Meisner}

Dept. of Gastrointestinal Surgery K, $\mathrm{H}: S$ Bispebjerg Hospital, University of Copenhagen, Copenhagen, Denmark.

\section{References}

${ }^{1}$ Ell C, Remke S, May A et al. The first prospective controlled trial comparing wireless capsule endoscopy with push enteroscopy in chronic gastrointestinal bleeding. Endoscopy 2002; 34:685-689

${ }^{2}$ Lingenfelser T, Ell C. Lower gastrointestinal bleeding. Best Pract Res Clin Gastroenterol 2001; 15: 135-153

${ }^{3}$ Costamagna G, Shah SK, Riccioni ME et al. A prospective trial comparing small bowel radiographs and video capsule endoscopy for suspected small bowel disease. Gastroenterology 2002; 123: 999- 1005

${ }^{4}$ Lewis BS, Swain P. Capsule endoscopy in the evaluation of patients with suspect small intestinal bleeding: results of a pilot study. Gastrointest Endosc 2002; 56: $452-456$

${ }^{5}$ Mark RJ, Poen JC, Tran LM et al. Angiosarcoma: a report of 67 patients and a review of the literature. Cancer 1996; 77: $2400-2406$

\section{Corresponding Author}

\section{F. K. Knop, M.D.}

Dept. of Gastrointestinal Surgery K $\mathrm{H}: S$ Bispebjerg Hospital, University of Copenhagen, Bispebjerg Bakke 23 2400 Copenhagen NV, Denmark

Fax: $\quad+45-3531-6475$

E-mail: filipknop@dadlnet.dk

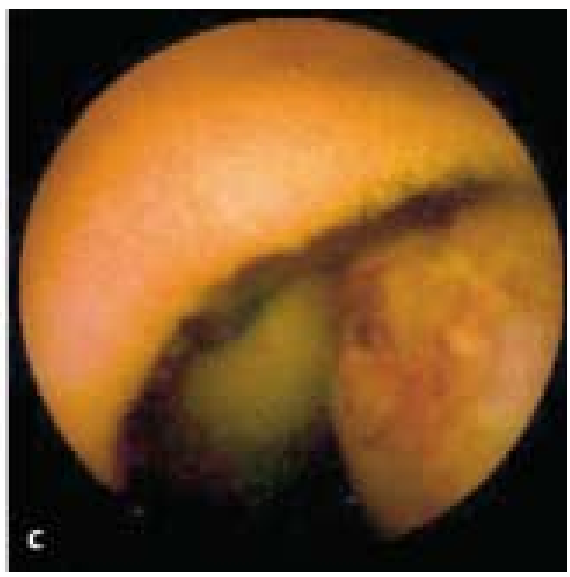

Figure 1 Capsule endoscopy images. a Normal jejunum with normal villi. b The dark and bloody liquid characterizing the intraluminal space beyond the tumor. c The protruding tumor, with a centrally seeping lesion in a blood vessel, as seen on the retrospective review. 